\title{
Duplicity Among Young Stars in Chamaeleon
}

\author{
WEN PING CHEN \& J. A. GRAHAM \\ Dept. of Terrestrial Magnetism, Carnegie Institution of Washington, \\ 5241 Broad Branch Road, NW, Washington, DC 20015, USA
}

\begin{abstract}
We present infrared imaging results of young stars in Cha I and II clouds that show either extended structure or a nearby neighboring star. Both regions appear to show high incidence rates of pairs. After excluding possible background stars, as judged from their brightness, color, or the local stellar surface number density, a frequency of $10 \%$ is deduced for binaries in Cha I with separations 2-5", which is comparable to that in Taurus and to that of main sequence field stars.
\end{abstract}

\section{INTRODUCTION}

Various detecting techniques have been applied in the past few years to the discovery of pre-main sequence (PMS) binaries, and the list of identified systems has been steadily accumulating. Taurus is the only region that has been surveyed systematically for PMS binaries thus far, and the binary frequency in the cloud has been found to be at least comparable to that of the main sequence field population over several decades in period (see the articles by Ghez et al., Leinert et al., and Simon in this volume.)

With its proximity $(150 \mathrm{pc})$ and abundance of young stars, the Chamaeleon region ( $\alpha \sim 11^{h} ; \delta \sim-77^{\circ}$ ) offers an excellent opportunity to collect a large sample of PMS binaries (see Schwartz 1991 for an overview of the region.) Recent studies of the Chamaeleon I and II dark clouds are reported in Gauvin \& Strom (1991), Whittet et al. (1991), and Prusti et al. (1992). Binaries identified in Chamaeleon not only can add to the PMS binary statistics but allow a comparison of their characteristics with those in other star-forming regions.

\section{OBSERVATIONS AND RESULTS}

Infrared images of young stars in Cha I and II were obtained in March 1991 and February 1992 with an IR camera (Persson et al. 1992) on the Las Campanas $2.5 \mathrm{~m}$ duPont Telescope. The camera was equipped with a NICMOS2 $(128 \times 128$ elements) detector. In each run the seeing conditions were excellent and a plate scale of $0.25^{\prime \prime} /$ pixel was used. A total of 67 association members in Cha I and 19 in Cha II (from lists in Schwartz 1991) were observed with standard infrared $J, H$, and $K$ filters. The complete result of photometry and consequent study of young stellar population will be published elsewhere. Here we present data for stars that either have neighboring companions or show elongated structure in their images.

Table 1 lists the stars in Cha $I$ that show subarcsecond structure. The naming of stars follows that of Schwartz (1991). The PA specifies the position angle of the elongation. The combined fluxes are given as $J, H, K$ magnitudes. These binaries should be further observed with higher angular resolution such as by speckle interferometry to fully resolve the individual components. Resolved 
TABLE 1. Stars in Cha I with Extended Structure ( $\left.\leqslant 1^{\prime \prime}\right)$

\begin{tabular}{|lcrrcc|}
\hline Star & PA & $J$ (mag) & $H$ (mag) & $K$ (mag) & Remarks \\
\hline T24 & S & 10.99 & 9.95 & 9.40 & see also Table 2 \\
T27 & N & 10.56 & 9.92 & 9.59 & \\
T31 & S & 8.49 & 7.69 & & sat. at $K$ \\
T43 & NS & 11.27 & 9.99 & 9.24 & \\
C1-18 & SW & 11.95 & 10.37 & 9.38 & high concentration of stars \\
\hline
\end{tabular}

binary/multiple systems in Cha I with separation less than $20^{\prime \prime}$ are summarized in Table 2. The angular separation and position angle of the companion are given, along with its magnitude differences with respect to the primary (defined as the brighter component at $K$ ). Table 3 lists the 5 pairs in Cha II with separations less than $5^{\prime \prime}$.

In Cha I, 15 of the 67 stars (22\%) observed are found to have at least one neighboring star within about $0 " 8$ to $5-6^{\prime \prime}$. Cha I also shows a high incidence rate of wide systems (between $5^{\prime \prime}-10^{\prime \prime}$ and $10^{\prime \prime}-20^{\prime \prime}$ ). Some of these neighboring stars are very faint or have infrared colors substantially bluer than those of the primaries, thus are likely background stars. An asterisk in last column indicates the binary/multiple system is situated in a region of high concentration of stars, as judged from mapping a large area around the field. These systems excluded, there are 4 systems with subarcsecond separations and 7 systems in Table 2 (T3, T6, T14a, T26, T39, T43, T51) with 2-5" companions. In particular, T39 (Sz30), seen against dark cloud, is noted as a triple system with both companions closer than $5^{\prime \prime}$. Cha II, with a small sample, also shows a large fraction (5 out of 19) of systems less than $5^{\prime \prime}$. In comparison to Taurus, which is at a similar distance, background confusion from galactic field stars is evidently more noticeable in Cha. Galactic models indicate (T. Jones, private communication) a star count at $\mathrm{K}$ band $(\mathrm{K} \varsigma 14 \mathrm{mag})$ toward Chamaeleon $\left(\ell \sim 300^{\circ} ; b \sim-15^{\circ}\right)$ to be at least twice of that toward Taurus $\left(\ell \sim 180^{\circ} ; b \sim-15^{\circ}\right)$.

The $10 \%$ frequency for $2-5^{\prime \prime}$ binaries in Cha $I$ is close to the $7 \%$ in Taurus (Chen 1990) within the same angular range and is comparable to that of main sequence field stars (Duquennoy \& Mayor 1991).

\section{REFERENCES}

Chen, W.P. 1990, PhD dissertation, SUNY-Stony Brook

Duquennoy, A. \& Mayor, M. 1991, $A \& A, 248,485$

Gauvin, L.S. \& Strom, K.M. 1992, ApJ, 385, 217

Persson, S.E., West, S.C., Carr, D.M., Sivaramakeishnan, A., \& Murphy, D.C. 1992, PASP, 104, 204

Prusti, T., Whittet, D.C.B., \& Wesselius, P.R. 1992, MNRAS, 254, 361

Schwartz, R.D. 1991, in Low Mass Star Formation in Southern Molecular Clouds, ed. B. Reipurth, ESO Scientific Report, No. 11, p. 93

Whittet, D.C.B., Assendorp, R., Prusti, T., Roth, M., \& Wesselius, P.R., 1991, A\&A, 251,524 
TABLE 2. Resolved Companions in Cha I

\begin{tabular}{|c|c|c|c|c|c|c|}
\hline Star & $\begin{array}{c}\mathbf{R} \\
\left({ }^{\prime \prime}\right)\end{array}$ & $\begin{array}{l}\text { PA } \\
\left({ }^{\circ}\right)\end{array}$ & $\underset{(\mathrm{mag})}{\Delta \boldsymbol{J}}$ & $\begin{array}{c}\Delta \boldsymbol{H} \\
\text { (mag) }\end{array}$ & $\underset{\text { (mag) }}{\Delta K}$ & Remarks \\
\hline T3 & 2.1 & 288 & 0.81 & 1.17 & 1.69 & \\
\hline \multirow[t]{2}{*}{ T6 } & 5.0 & 123 & 2.79 & 3. & 3.20 & \\
\hline & 11.8 & 313 & 3.5 & 4.26 & 4.35 & \\
\hline $\mathbf{T} 12$ & 8.2 & 335 & 2.12 & 1.94 & 2.06 & \\
\hline $\mathrm{T} 14 \mathrm{a}$ & 2.5 & 53 & 1.89 & 1.25 & 1.12 & \\
\hline $\mathrm{T} 17$ & 12.7 & 21 & 4.18 & 3.9 & 4.35 & $*$ \\
\hline T19 & 10.2 & 28 & 1.24 & 0.68 & 0.42 & \\
\hline \multirow[t]{2}{*}{ T24 } & 10.0 & 77 & 4.5 & 5.1 & 5.3 & may be nonstellar \\
\hline & 11.9 & 250 & 5.1 & 5.2 & 6.1 & may be nonstellar \\
\hline \multirow[t]{3}{*}{$\mathrm{T} 26$} & 4.5 & 203 & & & & * saturated \\
\hline & 9.3 & 2 & & & & * saturated \\
\hline & 9.3 & 180 & & & & * saturated \\
\hline $\mathrm{T} 30 / 31$ & 16.2 & 221 & 2.97 & 2.92 & & T31 extended \\
\hline T33 & 2.5 & 285 & -0.22 & 0.32 & 1.25 & \\
\hline T34 & 3.4 & 222 & & & $>5$ & faint \\
\hline \multirow[t]{2}{*}{ T39 } & 1.2 & 24 & 0.44 & 0.26 & 0.19 & \\
\hline & 4.4 & 70 & 0.79 & 0.73 & 0.67 & \\
\hline \multirow[t]{2}{*}{$\mathrm{T} 47$} & 11.7 & 162 & 0.62 & 1.19 & 1.53 & \\
\hline & 12.8 & 140 & 2.78 & 3.01 & 3.03 & \\
\hline \multirow[t]{2}{*}{ T51 } & 1.8 & 164 & 2.03 & 1.97 & 2.25 & \\
\hline & 10.9 & 246 & -0.07 & 0.01 & 0.31 & \\
\hline \multirow[t]{2}{*}{ T52/53 } & 11.0 & 100 & 3.19 & & & * T52 saturated \\
\hline & 10.9 & 213 & 5.8 & & & * T52 saturated \\
\hline T55 & 6. & NW & & & $>5$ & faint \\
\hline C1-18 & 9.9 & 93 & & 5.28 & 6.00 & * \\
\hline C1-15 & 11.1 & 321 & & & & * \\
\hline $\mathrm{C} 1-24 / 25$ & 18.6 & 51 & -1.61 & 0.59 & 0.39 & triple \\
\hline C2-5 & 5.4 & 63 & & & $>5$ & faint \\
\hline C7-1 & 5.7 & 216 & 3.02 & 2.76 & 2.89 & \\
\hline C7-11 & 13.5 & 252 & 4.8 & 5.26 & 5.25 & \\
\hline
\end{tabular}

TABLE 3. Stars in Cha II with Companions $\lesssim 5^{\prime \prime}$

\begin{tabular}{|lrrl|}
\hline Star & R (") & PA & Remarks \\
\hline T1 & 4 & S & \\
T3 & 2 & NE & \\
T14 & $<1$ & S & \\
T15 & 3 & E & \\
T17 & 1 & W & high concentration of stars \\
\hline
\end{tabular}

